\title{
SIMULATIONS OF PASSIVE MATRIX POLYMER IMAGE SENSORS
}

\author{
D. BRAUN *, G. YU ** \\ *EE Department, Cal Poly State University, San Luis Obispo, CA 93407, dbraun@calpoly.edu \\ **UNIAX Corporation, Santa Barbara, CA 93117, GangYu@Uniax.com
}

\begin{abstract}
Two-dimensional passive photodiode matrices are hardly useful for image sensing due to the crosstalk between pixels. This crosstalk makes it difficult to recover information from individual pixels. A switching unit attached to each sensing unit has been the common solution in image sensors (such as in CMOS sensors and in TFT-PiN a-Si photosensors). A novel organic photodiode with voltage-switchable photosensitivity was developed recently. Passive photodiode matrices made with such organic photodiodes can be used for image sensing applications. This circuit simulation study demonstrates an effective scheme to extract images from passive photodiode matrices, concluding that individual photodiode parameters determine the contrast and resolution of $\mathrm{N}$ by $\mathrm{M}$ image sensors.
\end{abstract}

\section{INTRODUCTION}

Much interest in polymer and organic light-emitting diodes and photo-diodes derives from their potential to deliver the next generation of flat and flexible image sensors and passive matrix displays [1-9]. The devices are relatively easy to make, because they consist of a photosensitive or electroluminescent layer sandwiched between an anode, usually transparent, and a cathode. A passive matrix image sensor or display results by patterning the anode into columns and the cathode into rows to form an array of pixels from the intersections between the cathode and anode electrodes. Applying a bias to one anode and the opposite bias to one cathode allows light detection or causes light emission from the pixel shared by the two electrodes. Organic photodetectors demonstrated recently have sufficiently high photosensitivity, low dark current and large dynamic range to allow demonstrations of large area photodiode arrays and page size image scanners. When employed in one-dimensional linear photodiode arrays or in two-dimensional active matrices, the sensors can distinguish gray levels with at least 12 bit resolution, which is attractive for high quality imaging applications. This study demonstrates a drive scheme that allows high resolution image sensing using a two-dimensional passive matrix configuration.

\section{THEORY}

A passive matrix image sensor consists of an array of photodiodes with devices in the same row sharing a common cathode electrode and devices in the same column sharing a common anode electrode. Figure 1 illustrates the electrical connections for a 4 by 4 pixel sensor. 


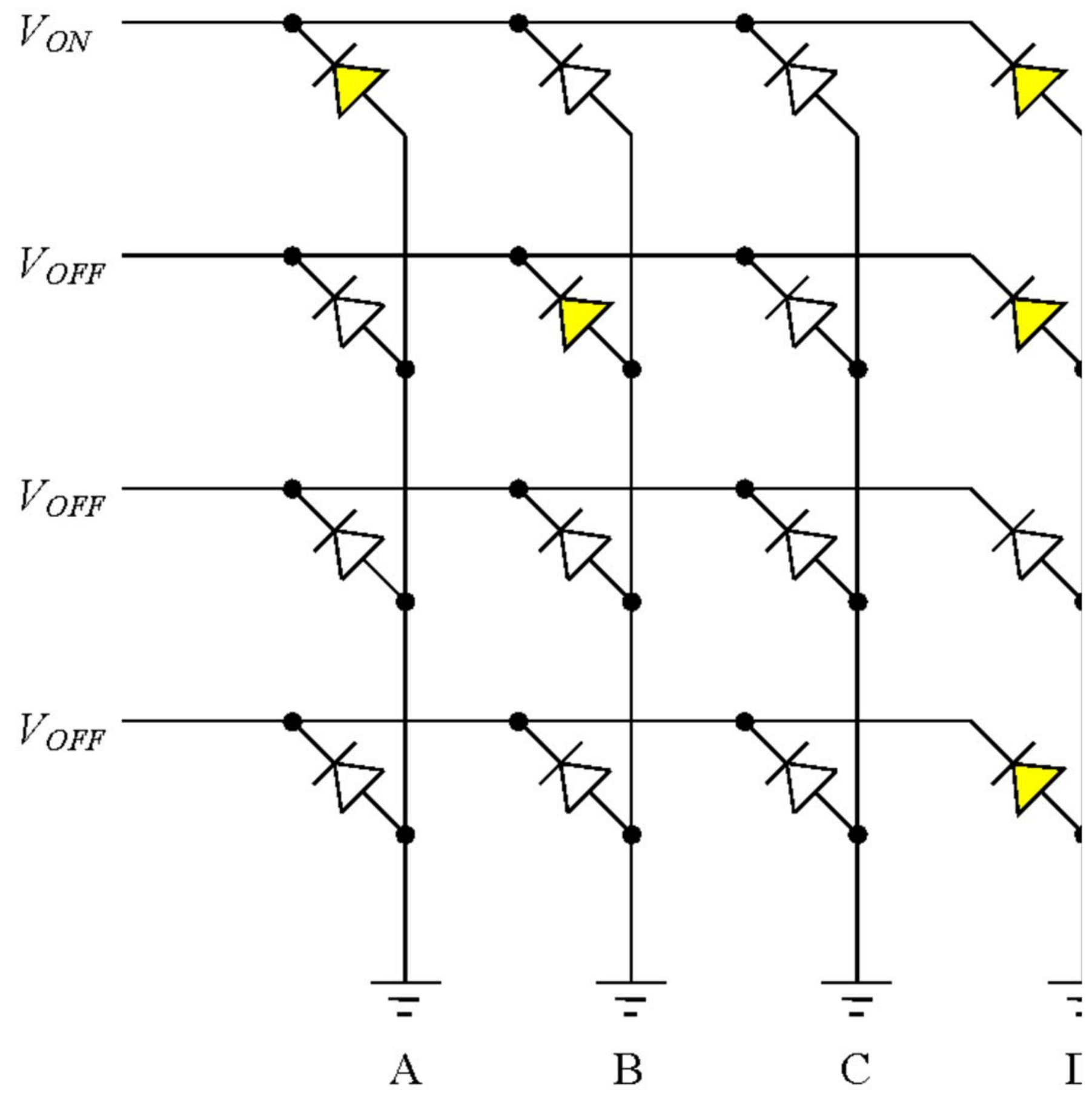

Figure 1 - Passive matrix array of photo-diodes. The rows form the cathode electrodes, and the columns form the anode electrodes. Yellow pixels are illuminated with $7 \mathrm{~mW} / \mathrm{cm}^{2}$.

In order to sense the photo-current of a given pixel, applying a positive voltage to a row electrode with respect to a column electrode will reverse bias the diode located at the intersection of the two electrodes and create a current dominated by the photo-current of the diode. In practice, other pixels in the same column contribute dark-current and photo-current to the column current. Consider the diodes in column A with the pixel in the top row illuminated by light and all other pixels dark. With a positive bias, $V_{O N}$, applied to the top row with respect to column A, the top pixel will generate photo-current. The current measured at the bottom of column A will include the photo-current due to the top pixel plus the sum of the current flowing in the other pixels. If the other pixels are biased to a voltage $V_{O F F}$, the total current measured at column $\mathrm{A}$ is 


$$
I_{A}=I_{L}\left(-V_{\text {ON }}\right)+(N-1) I_{D}\left(-V_{\text {OFF }}\right)
$$

where $I_{D}(V)$ is the current versus voltage characteristic of the dark photodiode, $I_{L}(V)$ is the current versus voltage characteristic of the illuminated photodiode, and $N$ is the number of rows. The negative signs indicate that a positive voltage applied to a row reverse biases the diodes in the row.

Equation 1 indicates a best case starting point to devise a read-out scheme for a row-scan multiplexed sensor, in which all pixels in non-addressed rows remain dark. With sufficiently conducting row electrodes, column currents flow in parallel and independently of other column currents, so it is possible to measure column currents simultaneously in order to sense the illumination incident on the addressed row. The ratio of the first term on the right hand side of equation 1 to the second term would then give the best case indication of the sensor's contrast, or how many gray levels the sensor could distinguish. The case that light also illuminates non-addressed rows provides a more conservative estimate of the sensor's ability to distinguish illuminated and non-illuminated pixels in an addressed row. In a column with the addressed pixel dark and one of the non-addressed pixels illuminated, equation 2 shows that the column current $I_{B}$ includes unwanted photo-current.

$$
I_{B}=I_{D}\left(-V_{O N}\right)+I_{L}\left(-V_{O F F}\right)+(N-2) I_{D}\left(-V_{O F F}\right)
$$

where any of the ( $N$ - 1) non-addressed rows could be illuminated. The degree by which the ratio of the currents $I_{A}$ to $I_{B}$ exceeds the number of rows indicates if it is possible to detect whether a given pixel is illuminated and how many shades of gray the sensor can distinguish. We define a figure of merit, $G$, to compare cases $A$ and $B$ and place a limit on the maximum number of gray levels a sensor can discriminate under various operating conditions:

$$
G=\frac{I_{A}}{(N-1) I_{B}}
$$




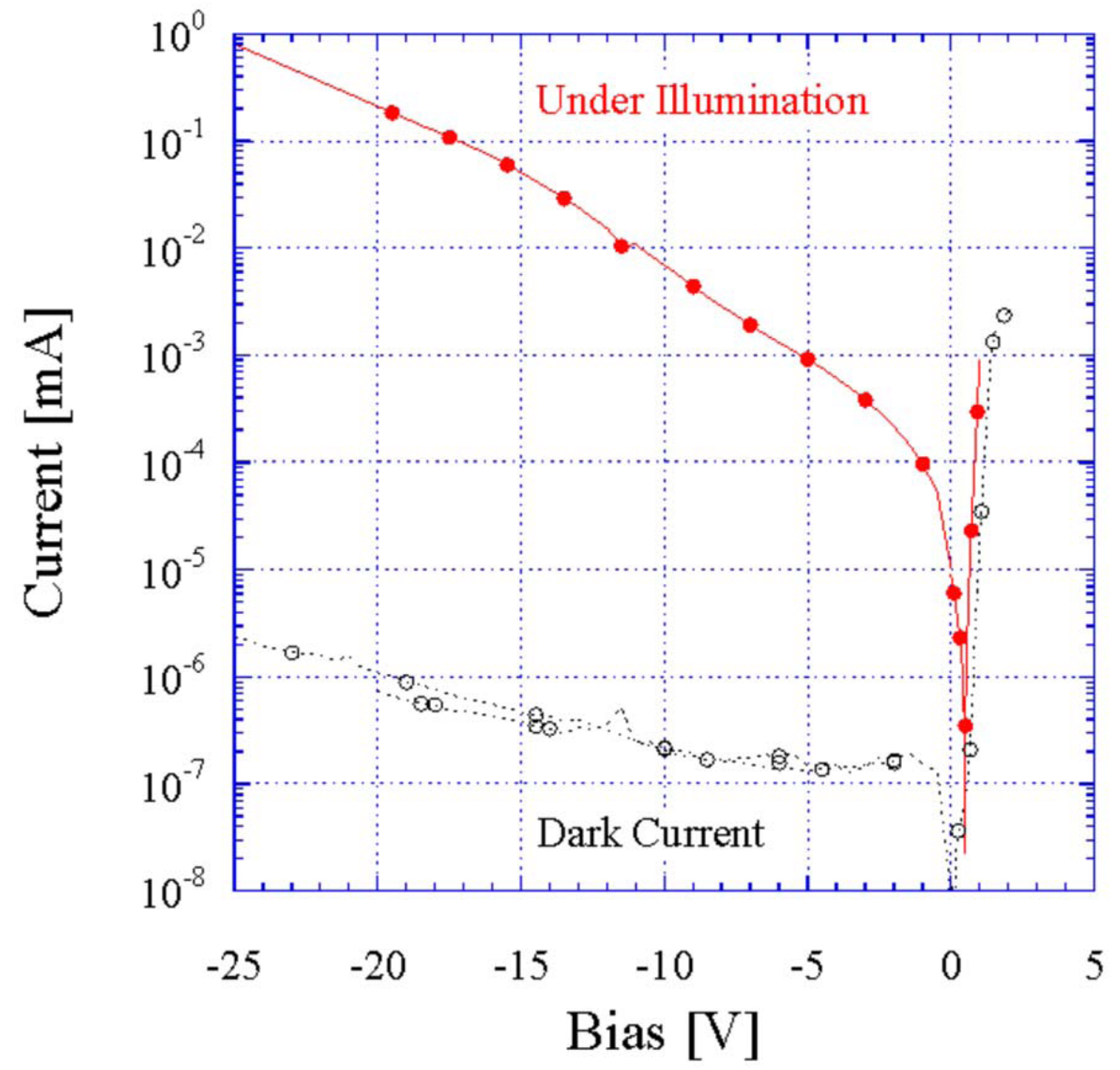

Figure $2 \mathrm{a}$ - Current versus voltage characteristics of $0.1 \mathrm{~cm}^{2}$ polymer photodiode in the dark (open circles) and illuminated by $7 \mathrm{~mW} / \mathrm{cm}^{2}$ (closed circles). 


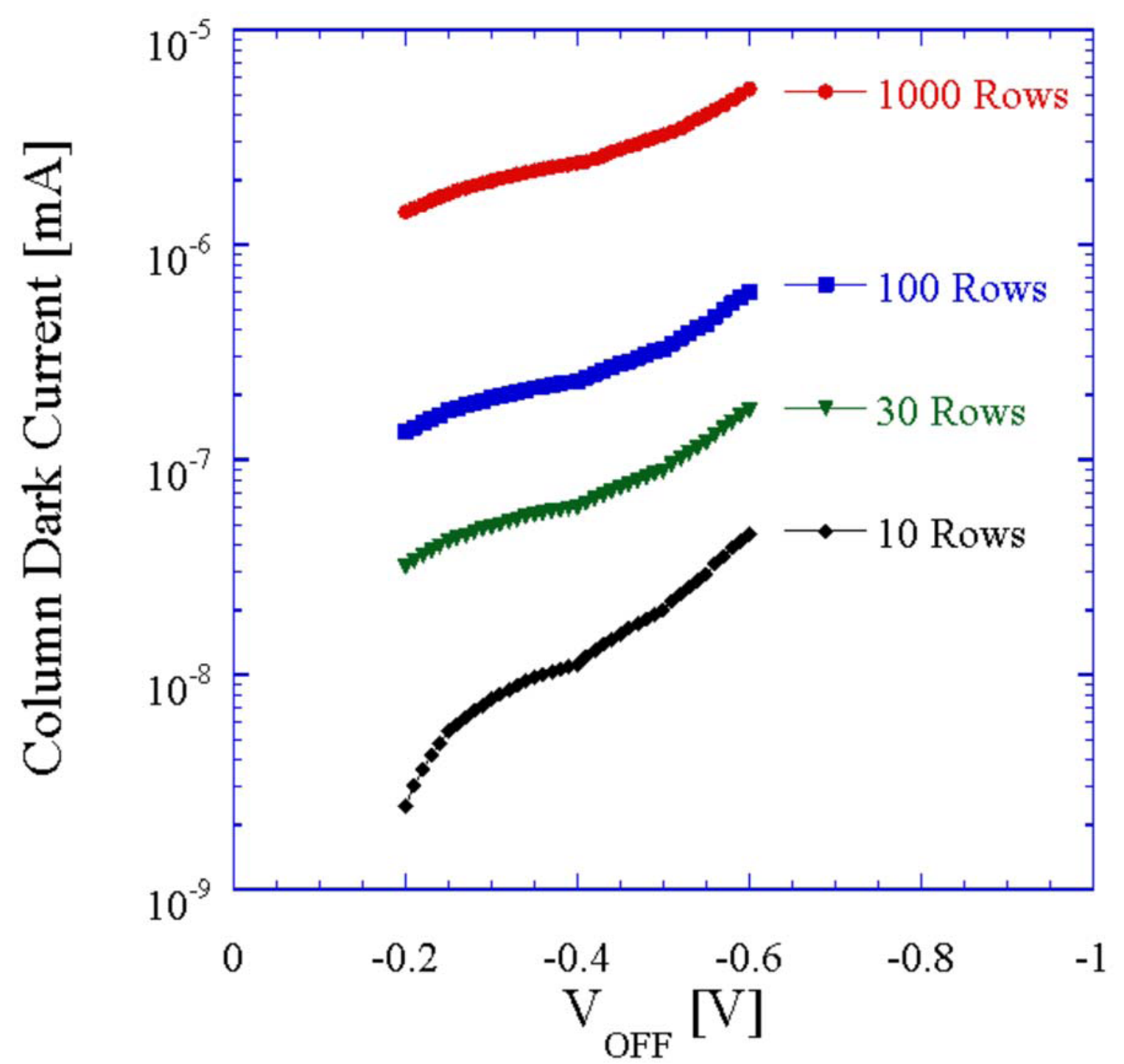

Figure $2 \mathrm{~b}$ - Current as a function of bias VOFF applied to the non-addressed rows in column $\mathrm{C}$ with all pixels in the column in the dark. $V_{O N}=10 \mathrm{~V}$.

\section{RESULTS}

The simulation results in figures 3, 4, and 5 use the current versus voltage characteristics shown in figure 2.[4-5] The approach used is to insert electrical data from figure 2 into a circuit simulation tool and enter a circuit net list consisting of a $N$ x $M$ array of $0.5 \mathrm{~mm}^{2}$ pixels connected as in figure 1.[10] Although the small currents minimize their impact, the simulations also take into account row and column electrode resistances.[11]

\section{Image Sensor Contrast}

Figure 3 plots the current detected from columns $\mathrm{A}$ and $\mathrm{B}$ in figure 1 as a function of $V_{O N}$ for $V_{O F F}=$ $-0.5 \mathrm{~V}$. These cases correspond to equations 1 and 2 . There is one lit pixel in each column, and each 
column is grounded. The figure shows that with a sufficient bias applied to the addressed row, significantly more current flows in column A with the lit pixel in the addressed row than in column B with the lit pixel in a non-addressed row. The current in column A containing the lit pixel in the addressed row increases exponentially with sense voltage, $V_{O N}$, as anticipated from the current versus voltage characteristics, because the photo-current in the lit pixel dominates the column current. For the same reason, this column current does not depend on display resolution for arrays having less than 100 rows. The current in column B containing the lit pixel in a non-addressed row remains roughly constant as a function of sense voltage, because the dark current does not depend strongly on reverse bias voltage. This current does increase with the number of rows, because it is dominated by the contributions from all of the non-addressed pixels.

Since figure 3 demonstrates that it is possible to distinguish which column contains an illuminated addressed pixel, equation 3 describes the maximum number of gray levels the sensor can distinguish. Figure 4 plots the maximum number of gray levels as a function of non-addressed row bias, $V_{O F F}$, showing that selecting $V_{O F F}$ to minimize the photo-current in non-addressed rows enables greater sensitivity. Figure 4 uses the absolute values of column currents to create the inflection voltage that emphasizes a sensor's maximum sensitivity to shades of gray. 


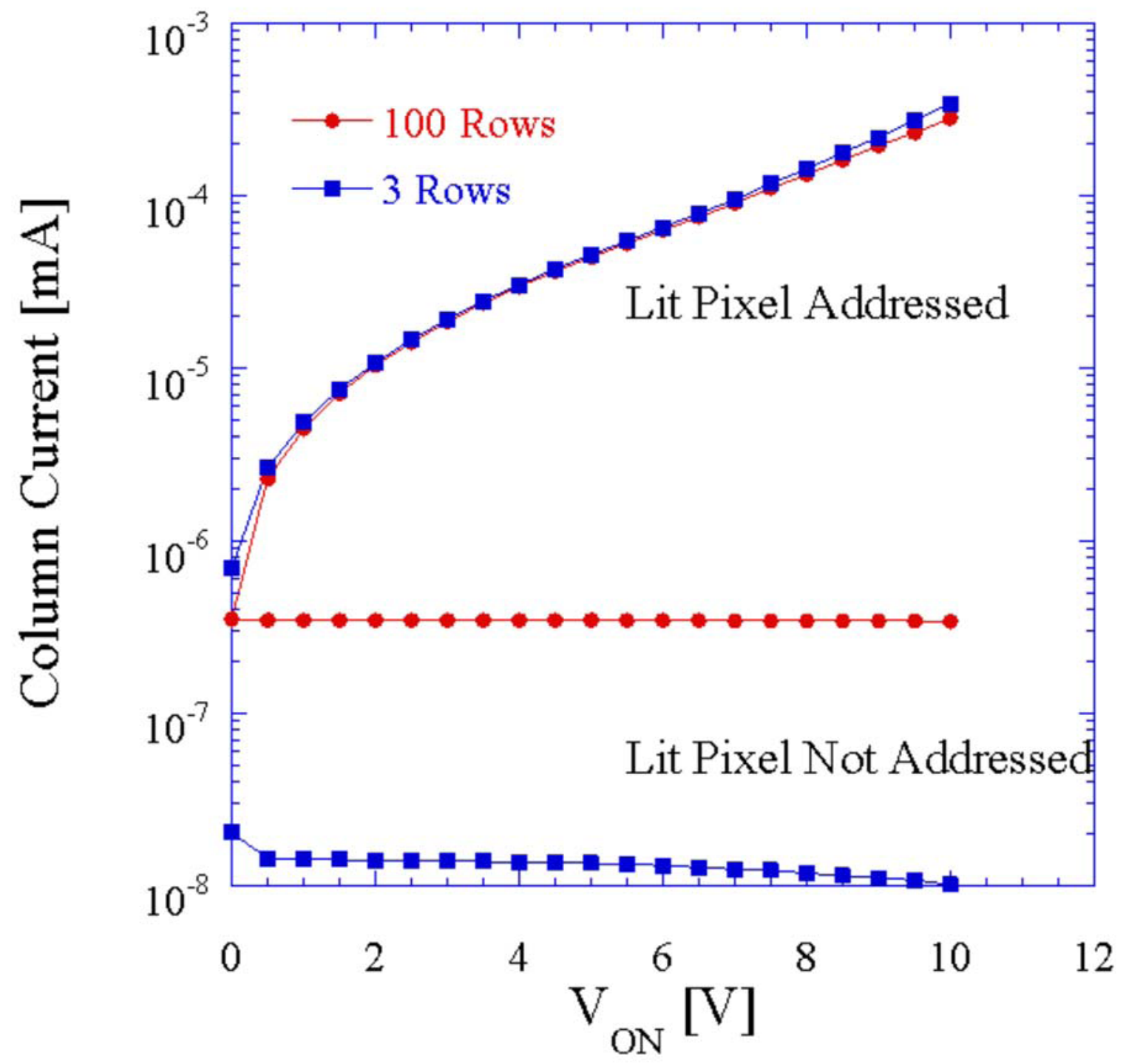

Figure 3 - Column currents versus addressing voltage, $V_{O N}$, at $V_{O F F}=-0.5 \mathrm{~V}$. 


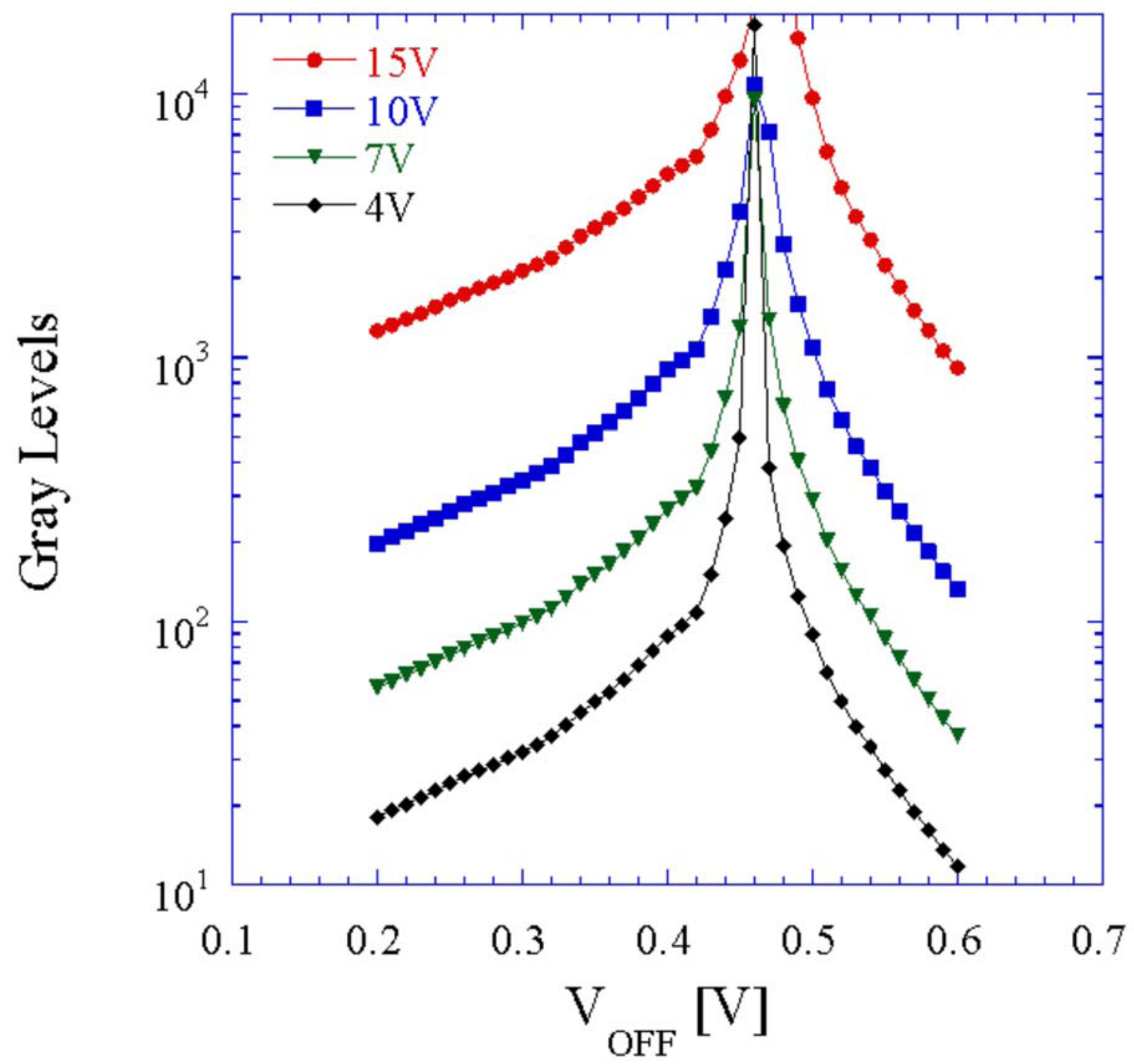

Figure 4 - Gray levels for sensor with 10 rows as a function of non-addressed row bias, VOFF, for several values of addressed row bias, $V_{O N}$. 


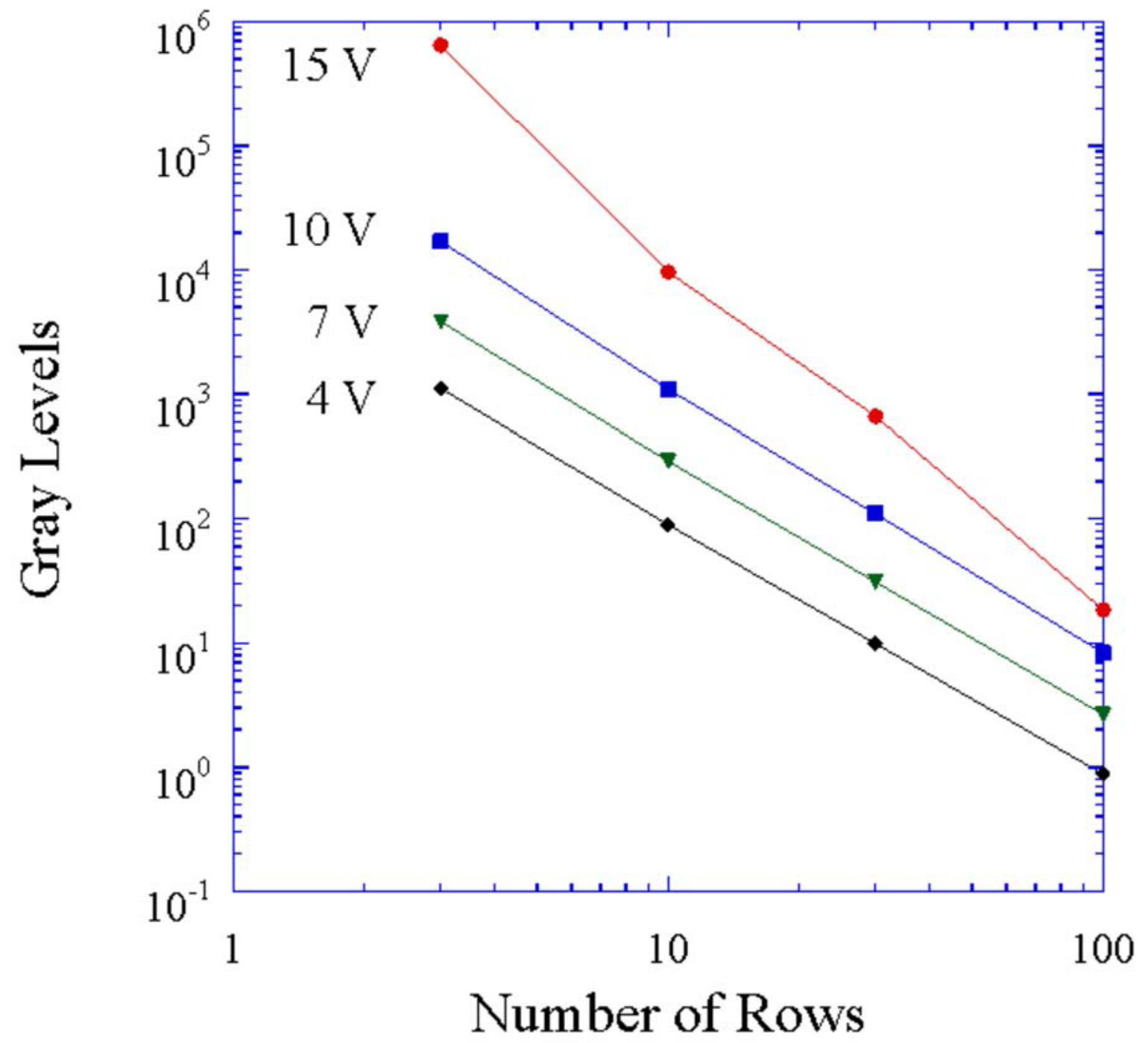

Figure 5 - Gray levels as a function of the number of rows of pixels in the matrix for several $V_{O N}$ from 4 $\mathrm{V}$ to $15 \mathrm{~V}$ with $V_{O F F}=-0.5 \mathrm{~V}$.

\section{$\underline{\text { Image Sensor Resolution }}$}

Figure 5 plots the ultimate number of gray levels a passive matrix polymer photodiode image sensor can distinguish as a function of the number of rows of pixels in the matrix. The figure applies equation 3 to the currents extracted from the circuit simulation results for several values of $V_{O N}$, with $V_{O F F}=-0.5 \mathrm{~V}$. Figure 5 illustrated the trends predicted by equations 1 - 3. A higher $V_{O N}$ increases the photocurrent $I_{L}$ in equation 1 , and the number of rows, $N$, appears in the denominator of equation 3 . Higher resolution requires more rows, so column currents increase, and it becomes more difficult to distinguish gray levels. In particular, the current $I_{B}$ limits the maximum number of gray levels that the sensor can distinguish. Also, a higher addressing voltage, $V_{O N}$, increases the desired photocurrent in an illuminated addressed pixel, so more gray levels become apparent. With appropriate selection of $V_{O N}$ and $V_{O F F}$, a polymer photodiode image sensor can achieve 12 bit gray scale resolution. 


\section{CONCLUSIONS}

Large area arrays of organic photodiodes provide an attractive approach for image sensing applications. Passive matrix image sensors based on organic photodiodes can achieve high sensitivity with relatively simple processing procedures that avoid the need to build a switching element into each pixel. Appropriate selection of row and column drive and sense voltages can take advantage of the current versus voltage characteristics of the photodiode to maintain sensitivity without the aid of a switching element associated with each pixel. This study used electrical circuit simulations to determine how the dark current and photo-current responses of the polymer photodiode influences the contrast and resolution of an image sensor. The simulation results show that optimizing the drive scheme can decrease the influence of unwanted current contributions from non-addressed pixels. A higher resolution image

sensor requires more pixels, but these undesirable currents increase with the number of pixels. Therefore, the contrast of the image sensor decreases with the number of pixels. Increasing the sense voltage, $V_{O N}$, applied to addressed pixels increases the desired photo-current from addressed pixels and increases the contrast of the sensor.

\section{REFERENCES}

1. C. W. Tang and S. A. VanSlyke, Appl. Phys. Lett. 51, p. 913-915 (1987).

2. J. H. Burroughes, D. D. C. Bradley, A. R. Brown, R. N. Marks, K. Mackay, R. H. Friend, P. L. Burns, and A. B. Holmes, Nature 347, p. 539-541 (1990);

R. H. Friend, R. W. Gymer, A. B. Holmes, J. H. Burroughes, R. N. Marks, C. Taliani, D. D. C. Bradley, D. A. Dos Santos, J. L. Brédas, M. Lögdlund \& W. R. Salaneck et al, Nature 397, p. 121-128 (1999).

3. D. Braun and A. J. Heeger, Appl. Phys. Lett. 58, p. 1982-1984 (1991);

G. Gustafsson, Y. Cao, G. M. Treacy, F. Klavetter, N. Colaneri, and A. J. Heeger, Nature 357, p. 477-479 (1992);

Y. Yang, MRS Bulletin 22, p. 31-38 (1997).

4. G. Yu , G. Srdanov, J. Wang and A. J. Heeger, Presentation B12.1, Spring Meeting of Materials Research Society, San Francisco, April 5 - 9 (1999).

5. G. Yu and Y. Cao, US Provisional Patent Application: 60/055/840 (Aug. 15, 1997).

6. G. Yu, J. Wang, J. McElvain, and A. J. Heeger, Adv. Mater. 10 \#17, p. 1431 (1998).

7. N.S. Sariciftci, L. Smilowitz, A.J. Heeger and F. Wudl, Science 258, p. 1474 (1992);

N.S. Sariciftci and A.J. Heeger, US Patent 5,333,183 (July 19, 1994);

N.S. Sariciftci and A.J. Heeger, US Patent 5,454,880 (Oct 3, 1995).

8. G. Yu, K. Pakbaz, and A.J. Heeger, Appl. Phys. Letters 64, p. 3422 (1994);

G. Yu, J. Gao, J.C. Hummelen, F. Wudl and A.J. Heeger, Science 270, p. 1789 (1995);

G. Yu and A.J. Heeger, J. Appl. Phys. 78, p. 4510 (1995).

9. J.J.M. Halls, C.A. Walsh, N.C. Greenham, E.A. Marseglia, R.H. Friend, S.C. Moratti and A.B. Holmes, Nature 376, p. 498 (1995).

10. D. Braun, Synth. Met. 92, p. 107-113 (1998).

11. D. Braun, J. Rowe, and G. Yu, Synth. Met. 102, p. 120-121 (1999). 